\title{
Ingested Foreign Body Causing Pylephlebitis Identified by Trans-abdominal Ultrasound
}

\author{
Paula Szanto, Tudor Vasile, Lidia Ciobanu, Doru Munteanu, Radu Badea \\ University of Medicine and Pharmacy, Iuliu Hatieganu, Cluj-Napoca, Romania, Regional Institute of Gastroenterology and Hepatology, \\ Cluj-Napoca, Romania
}

A 51-year-old male without significant medical history was admitted to the emergency department for two-week history. The patient reported a two-week history of fever, right upper quadrant abdominal pain, nausea and vomiting. He did not respond to empirical ampicillin treatment. He admitted a history of alcohol consumption and smoking. The clinical examination revealed mild jaundice, poor dentition and moderate right upper quadrant tenderness. Laboratory analysis detected leukocytosis with neutrophilia, thrombocytosis, iron deficiency anemia, mild elevation of direct bilirubin level and moderate cholestasis.

Abdominal ultrasound depicted portal vein thrombosis without any lesions of the biliary tract or other abdominal inflammatory processes. A 3-cm, linear hyperechoic structure was identified from the posterior duodenal wall, crossing perpendicularly through the portal vein (Figure 1). The foreign body was identified by anamnesis as a fish bone ingested one month previous of the onset of the symptoms. The results of computer tomography (CT) performed during the venous phase confirmed the presence of a fish bone as a foreign hyperdense structure as well as the portal vein thrombosis with an extensive perfusion defect in the liver parenchyma (Figure 2). No liver abscesses were identified.

The surgery was considered a high-risk procedure considering the site of the foreign body, crossing perpendicular through the portal vein. Conservative treatment with antibiotics and anticoagulants was initiated with rapid clinical improvement. At three months after the external the foreign body was at the same position, without clinical, biological or imagistic signs of portal vein thrombosis under the anticoagulative treatment (Figure 3). Written informed consent was obtained from the patient.

Fish bones are the most commonly ingested objects and the most common causes of foreign body perforation of the

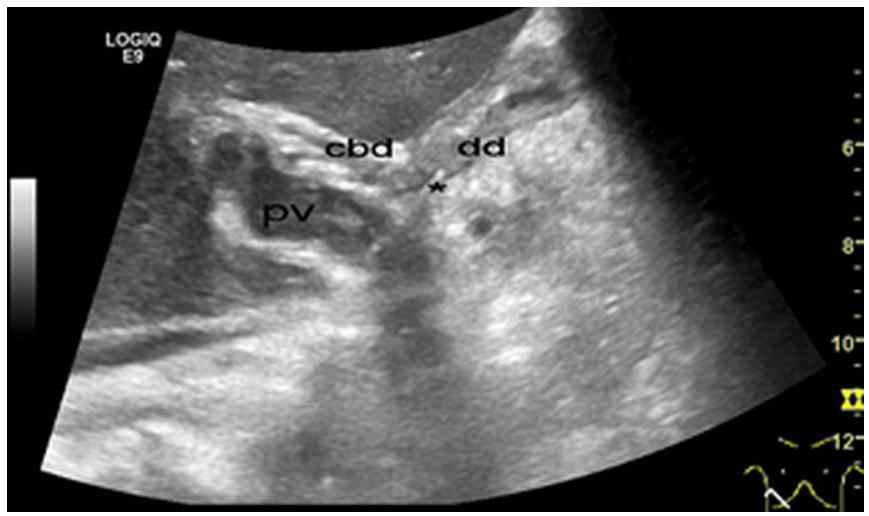

FIG. 1. Abdominal ultrasound focused on the liver hilum: a linear hyperechoic structure $\left(^{*}\right)$ was identified from the posterior duodenal (dd) wall, crossing perpendicular through the portal vein (pv). Above the portal vein the common bile duct (cbd) is imaged with normal size. A hyperechoic structure is identified within the portal vein, suggestive of thrombosis.

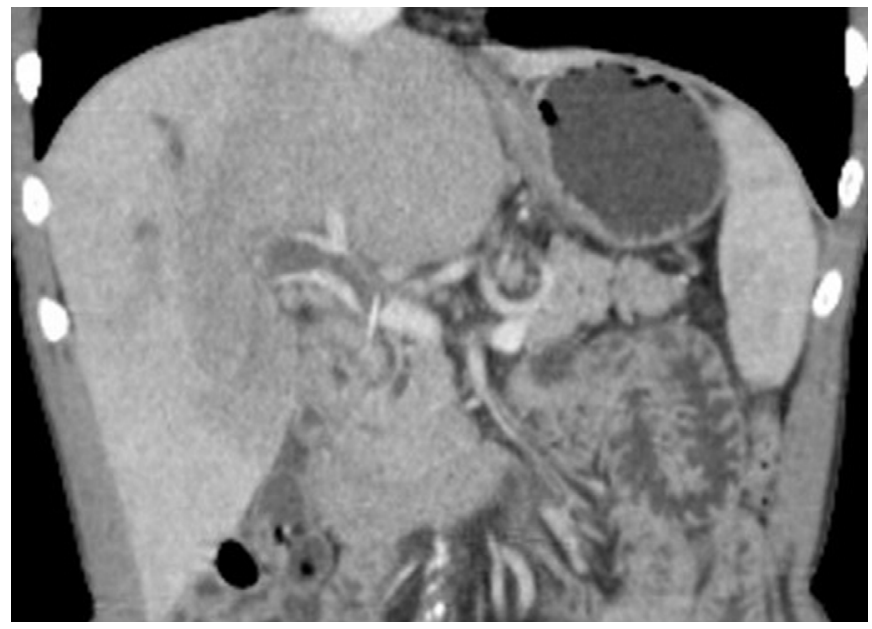

FIG. 2. Computer tomography: CT scan: venous phase: a foreign radioopaque structure crossing through the portal vein, with downstream thrombosis and extensive perfusion defect of liver parenchyma

Address for Correspondence: Dr. Lidia Ciobanu, University of Medicine and Pharmacy, Cluj-Napoca, Romania, Regional Institute of Gastroenteroloy and Hepatology, Cluj-Napoca, Romania 


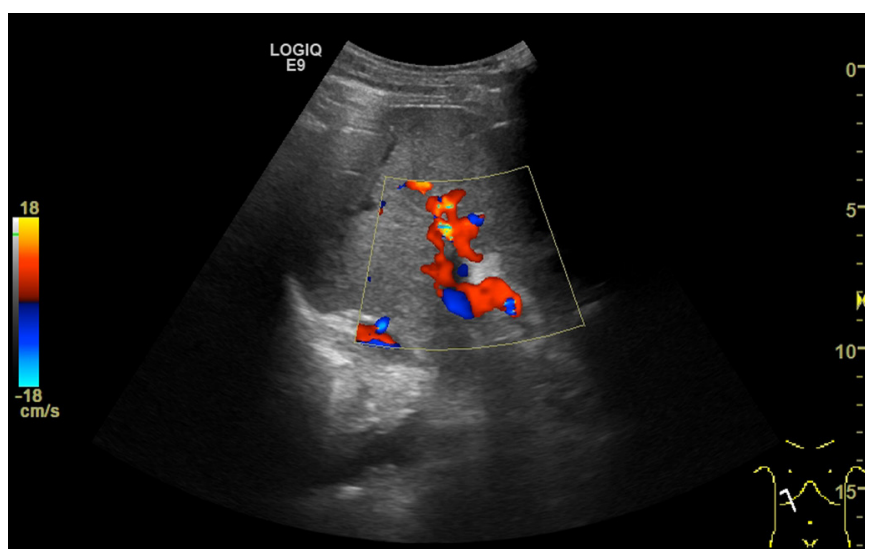

FIG. 3. Abdominal ultrasound with color Doppler technique at three months follow-up: blood flow present on portal vein.

gastrointestinal tract (GI tract), frequently occurring in regions of angulation (1) The diagnosis requires a high clinical suspicion because a wide diversity of clinical features could be present, and there may be a time lag of weeks or months from the time of ingestion to the onset of symptoms (1). Different others foreign bodies have been reported in literature to cause portal vein thrombosis: toothpick (2), intra-gastric migration of silicone gastric band (3), pancreatic stent migration (4).

The computer tomography scan is considered the gold standard for diagnosis. On CT scans, fish bones appear as calcified linear structures. The abdominal ultrasound represents an alternative for diagnosis since this technique is frequently requested as the first modality for the evaluation of abdominal pathology, and in many cases allowed a correct diagnosis to be reached without further assessment (5).

Ethics Committee Approval: N/A.
Informed Consent: Written informed consent was obtained from the patient.

Peer-review: Externally peer-reviewed.

Author contributions: Concept - P.S., T.V., R.B.; Design - P.S., T.V., L.C.; Supervision - P.S., L.C., R.B.; Resource - T.V., L.C., D.M,; Materials - P.S., T.V., D.M.; Data Collection and/or Processing - T.V., L.C., D.M.; Analysis and/or Interpretation - T.V., L.C., D.M.; Literature Search - P.S., T.V., L.C.; Writing - T.V., L.C.; Critical Reviews - P.S., R.B.

Conflict of Interest: No conflict of interest was declared by the authors.

Financial Disclosure: The authors declared that this study has received no financial support.

\section{REFERENCES}

1. Goh BK, Tan YM, Lin SE, Chow PK, Cheah FK, Ooi LL, et al. CT in the preoperative diagnosis of fish bone perforation of the gastrointestinal tract. AJR Am J Roentgenol 2006;187:710-4. [CrossRef]

2. Peters TG, Locke JR, Weight GR. Suppurative pylephlebitis caused by toothpick perforation. South Med J 1988;81:414-5. [CrossRef]

3. De Roover A, Detry O, Coimbra C, Hamoir E, Honoré P, Meurisse M. Pylephlebitis of the portal vein complicating intragastric migration of an adjustable gastric band. Obes Surg 2006;16:369-71. [CrossRef]

4. Leung WD, Parashette KR, Molleston JP, Sherman S. Pancreatic stent migration into the portal vein causing portal vein thrombosis: a rare complication of a prophylactic pancreatic stent. Pancreatology 2012;12:463-5. [CrossRef]

5. Coulier B. [Diagnostic ultrasonography of perforating foreign bodies of the digestive tract]. J Belge Radiol 1997;80:1-5. French. 